\title{
Estimation Techniques and Simulation Platforms for 77 GHz FMCW ACC Radars
}

\author{
Ali Bazzi ${ }^{1}$, Camilla Krnfelt ${ }^{1}$, Alain Pden ${ }^{1}$, Thierry Chonavel $^{1}$, Philippe Galaup $^{1}$, and Frantz Bodereau ${ }^{2}$ \\ ${ }^{1}$ Institut TELECOM, TELECOM Bretagne, Lab-STICC, Technople Brest Iroise CS 83818, 29238 Brest Cedex 3, France. \\ e-mail: First-name.Family-namedtelecom-bretagne.eu \\ 2 Autocruise, a TRW Automotive branch, 29238 Plouzan, France. e-mail: Frantz.Bodereau@trw.com
}

the date of receipt and acceptance should be inserted later

\begin{abstract}
This paper presents two radar simulation platforms that have been developed and evaluated. One is based on the Advanced Design System (ADS) and the other on Matlab. Both platforms are modeled using homodyne front-end $77 \mathrm{GHz}$ radar, based on commercially available monolithic microwave integrated circuits (MMIC). Known linear modulation formats such as the frequency modulation continuous wave (FMCW) and three-segment FMCW have been studied, and a new variant, the dual FMCW, is proposed for easier association between beat frequencies, while maintaining an excellent distance estimation of the targets. In the signal processing domain, new algorithms are proposed for the three-segment FMCW and for the dual FMCW. While both of these algorithms present the choice of either using complex or real data, the former allows faster signal processing, whereas the latter enables a simplified front-end architecture. The estimation performance of the modulation formats has been evaluated using the Cramer-Rao and Barankin bounds. It is found that the dual FMCW modulation format is slightly better than the other two formats tested in this work. A threshold effect is found at a signal-to-noise ratio (SNR) of $12 \mathrm{~dB}$ which means that, to be able to detect a target, the SNR should be above this value. In real hardware, the SNR detection limit should be set to about at least $15 \mathrm{~dB}$.
\end{abstract}

\section{Introduction}

In 2001 the European Union member states set up the goal to halve the number of fatalities caused by road accidents by the year of 2010 as compared to the rate in 1998 [1]. Despite safety efforts, rates only decreased by $27 \%$. Nowadays, most accidents are partly caused by human error or too long reaction time on the part 
of the driver [2], thus calling for perception assistance. In this area, ACC (Adaptive Cruise Control) radars are expected to play an important role.

In 2005, the European Telecommunications Standards Institute (ETSI) did temporarily open the $24 \mathrm{GHz}$ band for Short-Range automotive Radars (SRR) [3]. However, since the $24 \mathrm{GHz}$ band is also used for other systems, e.g. radio astronomy and weather forecasting, this band will only be allowed for car radars until 2013 , when it is presumed that the hardware technology (MMICs, antennas, etc.) will be mature enough to enable the development and production of automotive radar modules at the $76-81 \mathrm{GHz}$ band. After 2013, two bands are permanently allocated in Europe: one at $76-77 \mathrm{GHz}$ for LRRs and another at $77-81 \mathrm{GHz}$ for ultra wide band (UWB) short-range radars [4].

There are several advantages in moving from 24 to $76-81 \mathrm{GHz}$ : smaller size and weight of the radar frontend, RF chip set integration on a single chip, resulting in reduced losses and assembly costs, improved distance resolution due to wider available bandwidth; and narrower antenna beam which results in a better angular resolution.

In this paper, we describe efforts to improve the functionality of the ACC system by simultaneously developing the modulation format, detection and estimation algorithms, radar simulation tools and radar architecture. This is primarily done by the development of two simulation platforms using ADS by Agilent Technologies and Matlab by Mathworks.
Other simulation platforms have previously been described in the literature, e.g. [5] where an all-Matlab simulator is proposed. This simulator deals with hardware design, algorithm testing and performance analysis. Another elaborated simulation platform using ADS is presented in [6] in order to simulate a phase-coded CW radar sensor. In our work, the ADS-based platform allows the co-simulation using an envelope simulator for the $77 \mathrm{GHz}$ radar front-end and a Data Flow simulator which controls the digital signal processing at baseband. It also facilitates a correct and detailed modelling of the included components and signal analysis functions, such as spectrum analysers. The simulation results obtained from the ADS-based platform is compared to results obtained from the Matlab-based platform, to ensure that correct and feasible results are obtained. Furthermore, the Matlab platform allows us to establish statistical studies as well as the implementation and thorough testing of the algorithms to be used to detect and identify the targets.

For the presented radar application, the Cramer-Rao lower bound [7] and the Barankin bound [8] are used to calculate lower bounds of the mean square error for the distance and relative velocity estimation of the detected targets. Then, the parameters estimated from data obtained after Fourier transformation is compared to the bounds. The interest of these bounds is twofold. First, they supply a limit to best achievable results for a given waveform in terms not only of variance of the estimators, but also of detection capability. Second, they can 
tell us how far a certain processing algorithm is from the bound, and whether it is worth looking for a better one or not.

This paper is arranged as follows: In section II we present system requirements and discuss the advantages and drawbacks of different frequency modulation formats. Further on, we introduce the Cramer-Rao and Barankin bounds to estimate the performance of the chosen modulation formats, and finally we present a comparison between the theoretically calculated bounds and the practical results. Section III lays on a first simulation platform developed using the ADS co-simulation features, and a second platform using Matlab, together with a description of the general architecture of the radar system used throughout this work. The results from the two simulation platforms are compared and discussed in Section IV. In Section V, we show the results from extensive simulations to compare the efficiency of two proposed FMCW waveforms. Finally, in section VI, we give a conclusion about the results presented in the paper.

\section{FMCW waveforms for multitarget detection}

For the detection and parameter estimation of targets, the radar modulation format is the most important consideration. In pulse doppler radars, the distance to the target is given by the measure of time that has elapsed from the instant of transmission to the instant of reception of the pulse.

For Frequency Shift Keying (FSK) radar, two (or more) continuous signals shifted in frequency are transmitted [9]. The signal returned after reflection by the target is mixed with the transmitted signal, and thereby we will obtain the Doppler frequency which allows the calculation of the relative velocity of the target. The phase difference among the different FSK levels determines the target distance. The main disadvantage of FSK radar is that it can not discriminate fixed targets along the road, since they imperatively have the same relative velocity with respect to the radar. Moreover, targets with a relative velocity of zero (that is the same relative velocity as the vehicle that carries the radar) will return a Doppler frequency of zero, which means that they are not detected.

The Frequency Modulated Continuous Wave (FMCW) principle is to send a continuous signal with a linear frequency modulation [9]. The down-converted signal is referred to as the beat frequency. By varying the linear frequency modulation (up slopes, down slopes, flats etc.), on distinct time intervals, several beat frequencies are obtained, and the distance and relative velocity data of the targets can readily be resolved. One advantage of FMCW over FSK radar is that, thanks to the modulation format, fixed targets with different distances return different beat frequencies, even if their relative velocity is the same; hence they can be detected separately. The same goes for the targets that have a null relative 
velocity: since their distances are not the same, their beat frequencies will be different, and thus the targets can be distinguished according to their distance. When it comes to signal processing, FMCW radar does not add any difficulties as compared to the FSK radar, but rather the challenge lies in keeping the modulation linear in order to correctly estimate the beat frequencies.

Another waveform is the digital FMCW, obtained by the combination of LFMCW and FSK Modulation [10]. It has many advantages, such as the high distance and relative velocity resolutions, but its main problem is the complexity of its generation.

Given the drawbacks of the FSK modulation format as discussed above, this work is based on FMCW modulation formats.

\subsection{FMCW}

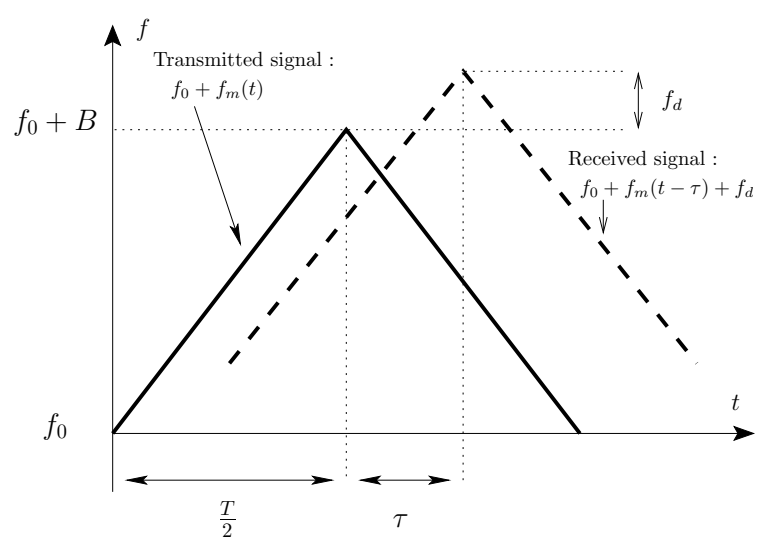

Fig. 1. Emitted FMCW waveform (solid line) and received waveform with delay and doppler offset (dashed line).

When using an FMCW modulation format, the target detection is obtained from the beat frequencies pre- sented for each target on the up and down frequency slopes, respectively. The beat frequencies arise from the following scheme: at a given transmission time, say $t$, the instantaneous frequency of the transmitted signal is $f_{0}+f_{m}(t)$. When this signal hits the target, it is shifted by the target's Doppler frequency, $f_{d}$. Once returned to the radar, the time $\tau=\frac{2 d}{c}$ has elapsed, that is, the time it took for it to travel to the target and back. Thus the signal returning from the target into the receiver at time $t^{\prime}=t+\tau$ is $f_{0}+f_{m}(t)+f_{d}$. This signal is mixed with the transmitted signal at that instant, that is $f_{0}+f_{m}(t+\tau)$. Written in another way, at time $t^{\prime}=t+\tau$, the transmitted signal with the frequency $f_{0}+f_{m}\left(t^{\prime}\right)$ is mixed with the received signal with frequency $f_{0}+f_{m}\left(t^{\prime}-\tau\right)+f_{d}$. This is illustrated in Fig. 1. Equation (1) gives the beat frequencies as a function of the target's relative velocity $v$ and distance $d$ [12] :

$$
\left\{\begin{array}{l}
f^{\text {up }}=\frac{2 v f_{0}}{c}-\frac{4 B d}{T c} \\
f^{\text {do }}=\frac{2 v f_{0}}{c}+\frac{4 B d}{T c},
\end{array}\right.
$$

where $c$ is the speed of light and $B$ the chirp bandwidth.

Once the beat frequencies have been detected, the distance $d$ to the target can be calculated using (2) and its relative velocity, according to (3):

$$
d=-\frac{T c}{8 B}\left(f^{\text {up }}-f^{\text {do }}\right)
$$

$$
v=\frac{c}{4 f_{0}}\left(f^{\mathrm{up}}+f^{\mathrm{do}}\right)
$$


It is important to notice that in some cases beat frequencies can be negative, that is, the complex signal phase decreases with time. Thus, it is a must to find the correct sign in order to correctly calculate the target's data. Fig. 2 shows the beat frequencies for the up ramp and down ramp as a function of distance (0-200 m) and relative velocity range $(-180$ to $+360 \mathrm{~km} / \mathrm{h})$. The relative velocity for approaching vehicles is defined as positive. Here we have set $B=600 M H z$. The horizontal plane in the graphs is the zero frequency plane; accordingly, every beat frequency that is found below the horizontal plane actually has a negative sign. When considering Fig. 2, one realizes that in-phase demodulation, i.e. using only the real component of the signal, should be enough in the majority of situations. Indeed, Fig. 2 shows that in most situations we have $f^{u p} \leq 0$ and $f^{d o} \geq 0$. Based on these assumptions, errors would occur for short distance and positive relative velocity (where $f^{u p} \geq 0$ ) or negative relative velocity (where $\left.f^{d o} \leq 0\right)$. For relative velocities considered here, we

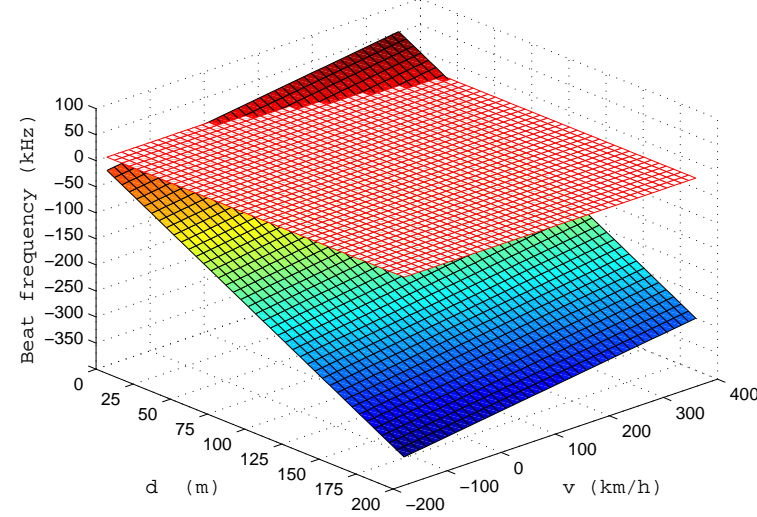

(a) Up ramp

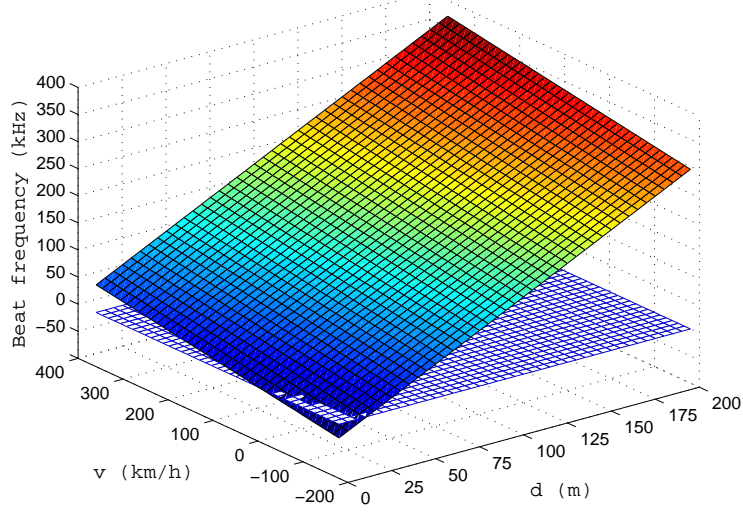

(b) Down ramp

Fig. 2. Beat frequencies as a function of distance and relative velocity. The beat frequency on the up ramp (a) is "negahave error-free situations when $f^{u p}(d, v)>\left|f^{u p}\left(0, v_{\max }\right)\right|_{\mathrm{ti}} \overline{\overline{\mathrm{e}}}$ " in most cases, while the down ramp (b) beat frequency is $\frac{2 f_{0} v_{\max }}{c}=f_{t}^{u p}$ and $f^{d o}(d, v)>f^{d o}\left(0,\left|v_{\min }\right|\right)=\frac{2 f_{0}\left|v_{\min }\right|}{c} \overline{\text { mstly }}$ "positive". The signs have to be taken into account in $f_{t}^{d o}$. When $f^{u p}$ and $f^{d o}$ are outside $\left[-f_{t}^{u p}, f_{t}^{u p}\right]$ and eq. (2) and (3).

$\left[-f_{t}^{d o}, f_{t}^{d o}\right]$ respectively, inphase demodulation is unambiguous. When $f^{u p} \in$ $\left[-f_{t}^{u p}, f_{t}^{u p}\right]$ and $f^{d o} \in\left[-f_{t}^{d o}, f_{t}^{d o}\right]$, ambiguity occurs. However, note that sign ambiguities mainly occur at short distances, implying potentially dangerous situations.

The FMCW format has some advantages over the FSK modulation, as discussed in the introduction. How- ever, it also suffers from drawbacks in a multi-target scenario. Indeed, every target presents a beat frequency on each ramp, and the association between the frequencies on the up ramp and the down ramp can be complicated, due to the fact that beat frequencies of targets can be ordered in a different way on up and down ramps. 
Consequently, more complex modulation formats such as the ones proposed in [10], [14] and [16] must be considered for multiple target detection. For these formats, simple distance criteria or more sophisticated ones [13] can be considered for the association of beat frequencies.

\subsection{Dual FMCW waveform}

\subsubsection{Presentation}

Reference [14] considers a waveform involving in two (or more) successive down ramps with slightly different slopes. The author shows that, for such a waveform, the order in which targets' beat frequencies are arranged on the first ramp is identical to their order on the second ramp. Thus, the association between the beat frequencies from different ramps is facilitated and the ambiguity of the simple FMCW is considerably alleviated. Unfortunately, this simple method of association has a problem, namely that it provides a bad estimate of relative velocity [14].

Nevertheless, the simplified association between beat frequencies is a great advantage of this waveform and was kept in mind in the design of a more advanced modulation format. Inspired by simple FMCW (which gives a good estimate of relative velocity) and the double down chirp modulation formats (with its beneficial beat frequency association), we propose a modulation format, which we call the "dual FMCW waveform". This modulation format involves two successive FMCW wave- forms with slightly different slopes on the first triangle as compared to the second triangle. The order of the beat frequencies of the targets is thus maintained between both up ramps as well as between both down ramps. Thus, the association of the beat frequencies is kept simple and the distance-velocity ambiguity is alleviated, while the variance of relative velocity estimation is kept small, thanks to the limited slope difference between the triangle ramps.

The structure of the dual FMCW waveform is summarized in Fig. (3). It is described by means of four parameters:

- The carrier frequency $\left(f_{0}\right)$

- The modulation bandwidth $(B)$

- The total duration of the modulation $(T)$

- The duration of the first triangle $(\theta)$

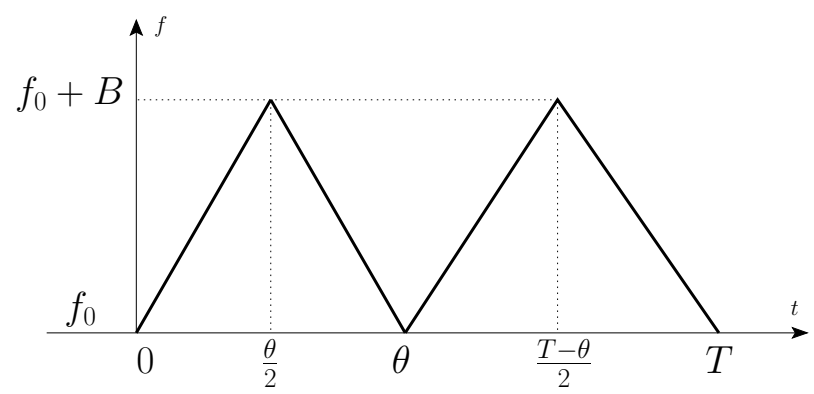

Fig. 3. Dual FMCW waveform.

\subsubsection{Parameter estimation for the dual FMCW}

waveform

For this dual FMCW waveform, we propose an algorithm where only the real part of the reflected signal 
is needed ${ }^{1}$. Thus, a simplified radar front-end architecture is also possible, compared to receivers with inphase and quadrature demodulation. Beat frequencies of targets are obtained by thresholding the periodograms [15] of the demodulated signals of each ramp. $f_{i}^{u p_{k}}$ denotes the $i^{t h}$ detected frequency on the $k^{t h}(k=1,2)$ up ramp. The parameter estimation is described hereafter. In the first step, since the order of targets is ensured only between both up ramps and between both down ramps, we calculate the distance and relative velocity using only up and down ramps respectively:

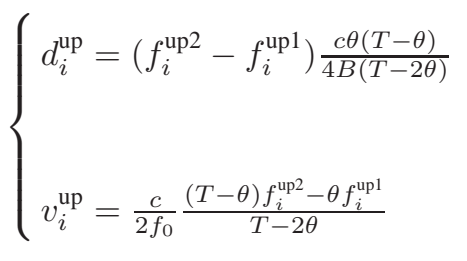

Up ramp estimations

$$
\left\{\begin{array}{l}
d_{i}^{\mathrm{do}}=\left(f_{i}^{\mathrm{do} 2}-f_{i}^{\mathrm{do} 1}\right) \frac{c \theta(T-\theta)}{4 B(2 \theta-T)} \\
v_{i}^{\mathrm{do}}=\frac{c}{2 f_{0}} \frac{(T-\theta) f_{i}^{\mathrm{do} 2}-\theta f_{i}^{\mathrm{do} 1}}{T-2 \theta}
\end{array}\right.
$$

Then, based on the rough estimation supplied by (4) and (5), we search matches between the positive distance and relative velocity estimates on the up ramps and those on the down ramps, This way, we can distinguish which of all beat frequencies are associated with each target. So, for a given target, we now know its beat frequencies on each of the four ramps. Then we

\footnotetext{
1 Note that for a spectrum based on real signals, we have to test twice as many beat frequencies on each ramp, as compared to a spectrum based on complex signals.
}

calculate an estimate for the first triangle (up and down ramps) and for the second triangle (up and down ramps):

$$
\left\{\begin{array}{l}
d=\left(f^{\mathrm{do} 1}-f^{\mathrm{up} 1}\right) \frac{c \theta}{8 B} \\
v=\left(f^{\mathrm{up} 1}+f^{\mathrm{do} 1}\right) \frac{\lambda}{4}
\end{array}\right.
$$

First triangle estimations

$$
\left\{\begin{array}{l}
d=\left(f^{\mathrm{do} 2}-f^{\mathrm{up} 2}\right) \frac{c(T-\theta)}{8 B} \\
v=\left(f^{\mathrm{up} 2}+f^{\mathrm{do} 2}\right) \frac{\lambda}{4}
\end{array}\right.
$$

Second triangle estimations

The final estimate is given by the mean of the estimates supplied by both triangles in (6) and (7). This algorithm, which provides us with the relative velocity at the targets, is summarized in Fig. 4.

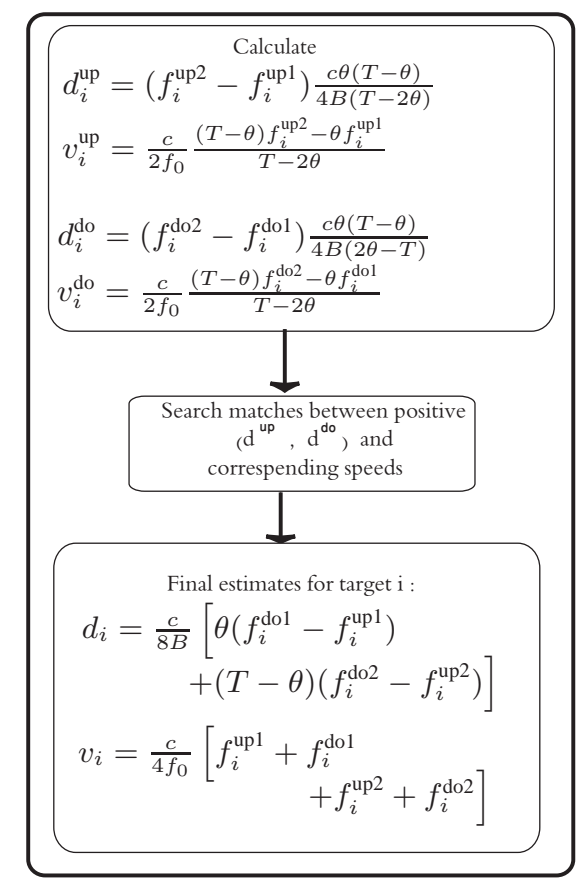

Fig. 4. Algorithm for calculating distance and relative velocity for dual FMCW waveform. 


\subsection{Three-segment FMCW waveform}

Another variant of the FMCW waveform was presented in [14], [16] and [17]. This modulation format has one part where the transmitted waveform is kept at a fix frequency. The rest of the waveform is composed of an up ramp and a down ramp. The echoes returned during the fixed part contain the Doppler frequencies, which provides us with the information on the targets' relative velocities. Moreover, the Doppler frequencies allow us to correctly associate the beat frequencies on the up and down ramps.

An example of the three-segment FMCW waveform is presented in Fig. 5, where $f_{0}, B$ and $T$ are kept equal to those of the double FMCW waveform. $\theta$ is set equal to $\frac{T}{3}$.

\subsubsection{Parameter estimation}

As for the case of the double FMCW, for the Threesegment FMCW waveform our algorithm allows the choice between the use of real or complex data processing (see footnote 1). Estimation is based on the fact that Doppler frequencies are given on the fixed frequency part of the modulation (hereafter called $f^{\text {pure }}$ ), thus providing the relative velocities of targets. Then, from the Doppler frequency information it is easy to find all possible corresponding Doppler-distance pairs on the up and down ramps. The distance estimation is given by (8) and (9).

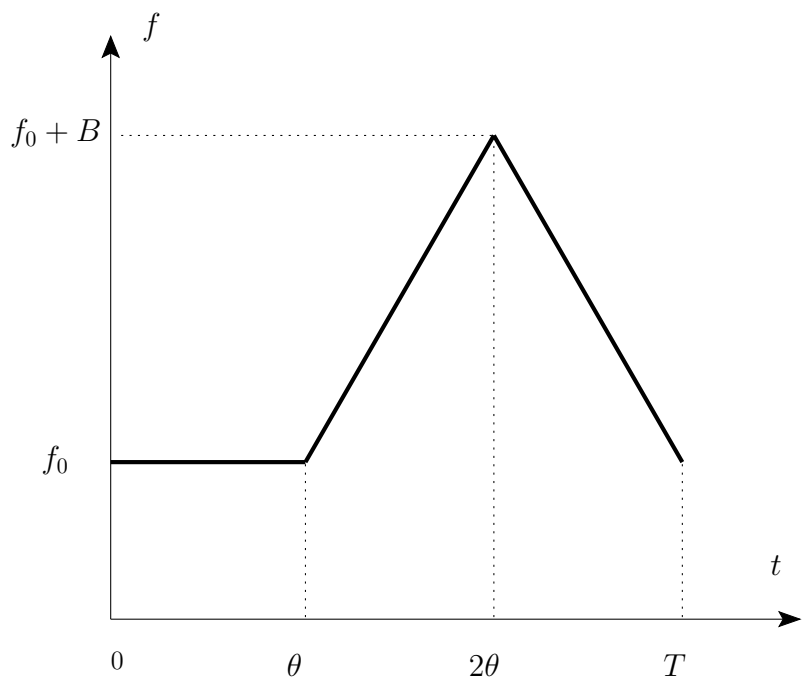

Fig. 5. Three-segment FMCW waveform.

$$
d_{i}^{\text {up }}=\left(f_{j}^{\text {pure }}-f_{k}^{\text {up }}\right) \frac{c \theta}{2 B}
$$

Up ramp distance estimation

$$
d_{i^{\prime}}^{\text {do }}=\left(f_{j^{\prime}}^{\text {do }}-f_{k^{\prime}}^{\text {pure }}\right) \frac{c \theta}{2 B}
$$

\section{Down ramp distance estimation}

Then, we seek the best possible match between the distances estimated on the up ramp and those estimated on the down ramp by minimizing $\sum_{i=1}^{I}\left(d_{i}^{\text {up }}-d_{\sigma(i)}^{\text {do }}\right)^{2}$ over all the permutations $\sigma($.$) of the set 1, \ldots, I$, where $I$ is the number of targets. The association thus made, the relative velocity and distance estimates of the targets can be performed using the associated beat frequencies inside the following equations:

$$
\left\{\begin{array}{l}
d=\left(f^{\text {do }}-f^{\text {up }}\right) \frac{c \theta}{4 B} \\
v=\left(f^{\text {up }}+f^{\text {do }}\right) \frac{c}{4 f_{0}}
\end{array}\right.
$$

A summary of this algorithm is shown in Fig. 6. 


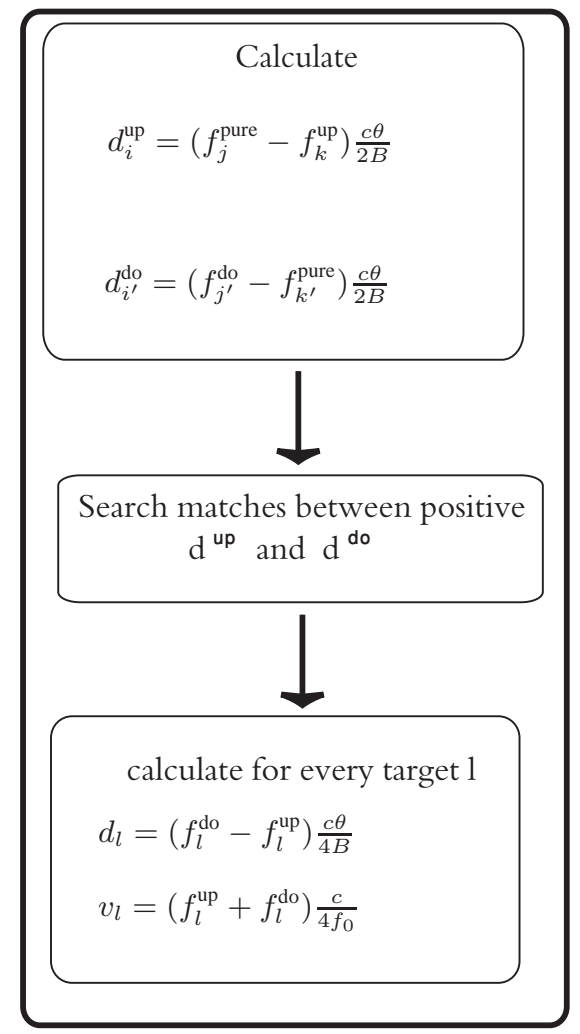

Fig. 6. Algorithm of calculating distance and relative velocity for Three-segment FMCW waveform.

\subsection{Performance Analysis}

In order to further decide which modulation format is best, a performance analysis is carried out. This kind of analysis helps in determining the standard deviation of the distance and the relative velocity as a function of the signal to noise ratio (SNR).

The searched parameters (distance, relative velocity, etc.) are calculated from the returned down converted baseband signal. This signal is always embedded in noise and thus the parameters can not be determined exactly, but have to be estimated.

To evaluate the performance of this estimate, a statistical bound, which is a minimal bound on the mean square error of any estimate, is used. The estimate is calculated as a function of SNR. In this work, the CramerRao lower bound, [7], and the Barankin bound, [8], [18] and [19], are considered.

The Cramer-Rao lower bound expresses a bound on the variance of estimators of a deterministic parameter. The bound states that the variance of any unbiased estimator is at least as high as the inverse of the Fisher information matrix [7]. Any lower bound represents a Mean Square Error (MSE) that is below the MSE of any possible estimator. Thus, the higher the lower bound, the better it characterizes the performance of a system.

The bound proposed by Barankin is higher than the Cramer-Rao lower bound for low SNR values. For high SNR values, it approaches the Cramer-Rao bound. In fact, the Cramer-Rao bound can be seen as a particular case of the Barankin bound, where the test points used are the only true parameters. The Barankin bound contains more information: it takes into consideration the secondary lobes of the ambiguity function [9], whereas the Cramer-Rao bound only considers the information given by the main lobe. By accounting for possible false detections around sidelobe maxima at low SNR, the Barankin bound supplies information not only on estimation variance, but also on detection capability: as the SNR decreases, a non detection will occur more frequently, thus possibly resulting in a break of the shape of the variance bound curve. On another hand, if an unbiased estimator of a parameter $\omega$ exists, then there exists an unbiased estimator that reaches the Barankin 
bound. Thus, the Barankin bound is the best lower bound and in addition, it is achievable. For an unbiased estimation $\hat{\omega}$, the Barankin bound yields [19]:

$$
\sigma_{d}^{2}=\operatorname{var}\left(-\alpha_{1} f^{\mathrm{up} 1}+\alpha_{1} f^{\mathrm{do} 1}-\alpha_{2} f^{\mathrm{up} 2}+\alpha_{2} f^{\mathrm{do} 2}\right)
$$$$
\sigma_{v}^{2}=\operatorname{var}\left(\beta f^{\text {up1 }}+\beta f^{\mathrm{do} 1}+\beta f^{\text {up2 }}+\beta f^{\mathrm{do} 2}\right),
$$

$$
\operatorname{cov}(\hat{\omega}) \geq \mathbf{L}\left(\Omega-1^{T}\right) \mathbf{L}^{T}
$$

where $1=[1, \ldots, 1]^{T}$ is a vector with length $M$, and $\mathbf{L}$ is a $M \times M$ matrix defined by:

$$
\begin{aligned}
\mathbf{L} & =\left[\begin{array}{c}
\omega_{1}-\omega \omega_{2}-\omega \ldots \omega_{M}-\omega \\
\end{array}\right] \\
& =\left[\begin{array}{l}
\tau_{1}-\tau \tau_{2}-\tau \ldots \tau_{M}-\tau \\
\nu_{1}-\nu \nu_{2}-\nu \ldots \nu_{M}-\nu
\end{array}\right]
\end{aligned}
$$

where $\left(\omega_{i}\right)_{i=1, \ldots, M}$ is any set of test points and $\Omega$ is the $M \times M$ Barankin matrix. The entries of $\Omega$ are given by:

$$
\Omega_{k, l}=\mathbb{E}\left[L\left(y, \omega, \omega_{k}\right) L\left(y, \omega, \omega_{l}\right)\right]
$$

where

$$
L\left(y, \omega, \omega_{k}\right)=\frac{p\left(y \mid \omega_{k}\right)}{p(y \mid \omega)}
$$

and $p(y \mid \omega)$ is the likelihood of observation $y$, given the parameter vector $\omega$.

To achieve the Barankin bound, we must maximize the right side of the inequality (11). So, our aim is to find a way to obtain the maximum bound and to compare it with the Cramer-Rao bound.

$$
\begin{aligned}
& \sigma_{d}^{2}=\alpha_{1}^{2}\left(\sigma_{f_{\text {up } 1}^{2}}^{2}+\sigma_{f^{\mathrm{do} 1}}^{2}\right)+\alpha_{2}^{2}\left(\sigma_{f^{\text {up } 2}}^{2}+\sigma_{f^{\mathrm{do} 2}}^{2}\right) \\
& \sigma_{v}^{2}=\beta^{2}\left(\sigma_{f_{\text {up } 1}^{2}}^{2}+\sigma_{f^{\mathrm{do} 1}}^{2}+\sigma_{f_{\text {up } 2}^{2}}^{2}+\sigma_{f^{\mathrm{do} 2}}^{2}\right)
\end{aligned}
$$

where $\operatorname{var}($.$) stands for the variance and$

$$
\begin{aligned}
& \alpha_{1}=\frac{c \theta}{16 B}, \\
& \alpha_{2}=\frac{c(T-\theta)}{16 B}, \\
& \beta=\frac{c}{8 f_{0}} .
\end{aligned}
$$

because of the independence of the estimations from one ramp to the next.

For the three-segment FMCW waveform we repeat the same steps performed for the previous waveform. Here (10) yields :

$$
\begin{aligned}
& \sigma_{d}^{2}=\sigma_{\left(-\gamma f^{\mathrm{up}}+\gamma f^{\mathrm{do}}\right)}^{2} \\
& \sigma_{v}^{2}=\sigma_{\left(\zeta f^{\mathrm{up}}+\zeta f^{\mathrm{do}}\right)}^{2}
\end{aligned}
$$

with:

\subsubsection{Approximations}

$$
\gamma=\frac{c \theta}{4 B}
$$

For the dual FMCW waveform, we can derive from (6) and (7) that the variance $\sigma_{d}^{2}$ of $d$ and $\sigma_{v}^{2}$ of $v$ are:

$$
\beta=\frac{c}{4 f_{0}}
$$


and lead to the relations

$$
\begin{gathered}
\sigma_{d}^{2}=\gamma^{2} \sigma_{f^{\text {up }}}^{2}+\gamma^{2} \sigma_{f^{\mathrm{do}}}^{2} \\
\sigma_{v}^{2}=\zeta^{2} \sigma_{f^{\text {up }}}^{2}+\zeta^{2} \sigma_{f^{\mathrm{do}}}^{2}
\end{gathered}
$$

Then, the corresponding Cramer-Rao bounds for $d$ and $v$ can be derived easily from (17) for the dual FMCW as follows:

$$
\begin{aligned}
\operatorname{CRB}(d)= & \alpha_{1}^{2}\left(\operatorname{CRB}\left(f^{\text {up } 1}\right)+\operatorname{CRB}\left(f^{\mathrm{do} 1}\right)\right)+ \\
& \alpha_{2}^{2}\left(\operatorname{CRB}\left(f^{\text {up } 2}\right)+\operatorname{CRB}\left(f^{\mathrm{do} 2}\right)\right) \\
\operatorname{CRB}(v)= & \beta^{2}\left(\operatorname{CRB}\left(f^{\text {up } 1}\right)+\operatorname{CRB}\left(f^{\mathrm{do} 1}\right)+\right. \\
& \left.\operatorname{CRB}\left(f^{\text {up } 2}\right)+\operatorname{CRB}\left(f^{\mathrm{do} 2}\right)\right),
\end{aligned}
$$

where $\mathrm{CRB}($.$) is the Cramer-Rao bound, and \mathrm{CRB}(f)$ is given by [15]:

$$
\operatorname{CRB}(f)=\frac{\sigma^{2}}{\sum_{n=0}^{N-1}\left(\frac{\partial s(n)}{\partial f}\right)^{2}},
$$

where $(s(n))_{n=0, \ldots, N-1}$ is the sampled signal with frequency $f$ and $\sigma^{2}$ is the variance of the noise.

For the three-segment FMCW, (20) leads to:

$$
\begin{aligned}
& \operatorname{CRB}(d)=\gamma^{2} \operatorname{CRB}\left(f^{\text {up }}\right)+\gamma^{2} \operatorname{CRB}\left(f^{\text {do }}\right) \\
& \operatorname{CRB}(v)=\zeta^{2} \operatorname{CRB}\left(f^{\text {up }}\right)+\zeta^{2} \operatorname{CRB}\left(f^{\text {do }}\right)
\end{aligned}
$$

As far as Barankin bounds are concerned, the analytical formulas for fixed test points have been obtained by using Mathematica software. Since they are very complicated, their expression is omitted here.

Thus, from (17) and (20), we can calculate the standard deviation (std) of the distance and relative velocity errors from the standard deviations of beat frequencies.

\subsubsection{Numerical Illustrations}

In Fig. 7 and Fig. 8 we display the standard deviation curves of both waveforms, for the distance and relative velocity error respectively, versus SNR. The standard deviation curves plotted here are those of periodogrambased estimations and Barankin and Cramer-Rao bounds. We can see that for low SNRs, the Barankin bound is far above the Cramer-Rao bound, and it is more in accordance with simulation results. When the SNR is high, the Barankin bound reaches the Cramer-Rao bound, and both bounds are close to the periodogram performance. In practice, there is a strong threshold effect around 12 $\mathrm{dB}$ both for distance and relative velocity estimations. Below the threshold, performance is very poor. This expresses the fact that at low SNRs, false detection often occurs. The threshold effect also appears on Barankin bounds, but at lower SNRs (around $5 d B$ ). This shows that periodogram-based estimators are quite far from optimal.

For both distance and relative velocity estimation, the performance of the dual FMCW waveform is slightly better than that of the three-segment FMCW waveform.

For the final calculation of the relative velocity from the three-segment waveform, we do not use the pure frequency. An optimisation based on the mean square error shows that the best results are obtained when taking into consideration the up and down beat frequencies only. Also, in Fig. 9, we can see that the absolute value of the beat frequency of the pure sinusoid part of the waveform is much lower in general than those of 


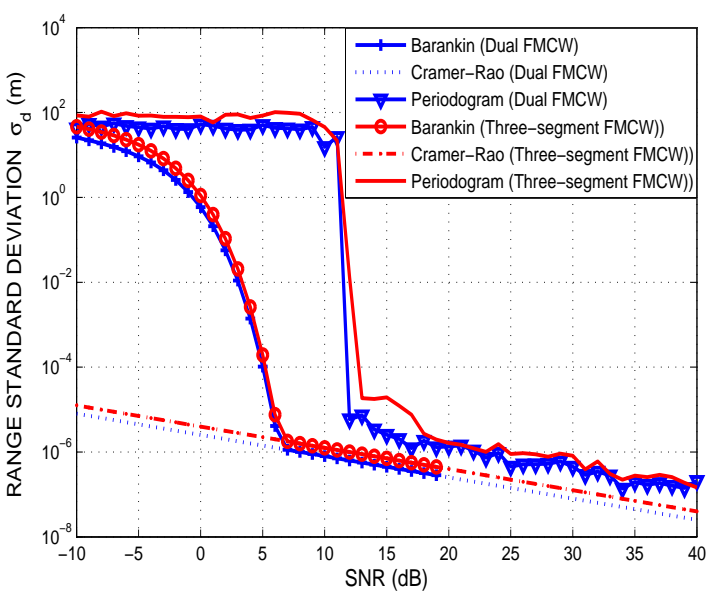

Fig. 7. Dual FMCW and Three-segment FMCW distance peformance $(d=50 \mathrm{~m}, v=80 \mathrm{~km} / \mathrm{h})$.

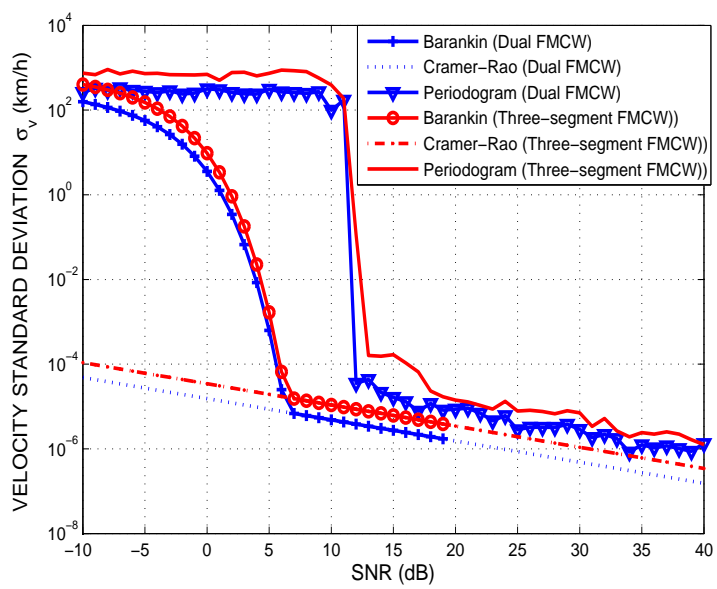

Fig. 8. Dual FMCW and Three-segment FMCW relative velocity performance $(d=50 \mathrm{~m}, v=80 \mathrm{~km} / \mathrm{h})$.

up and down ramps, resulting in a lower SNR (due to higher mixer noise at low frequencies), and thus in significantly higher variance of the estimator. Moreover, since the SNR of $f_{\text {pure }}$ is lower, it risks being closer to the threshold, and it is safer not to use it.

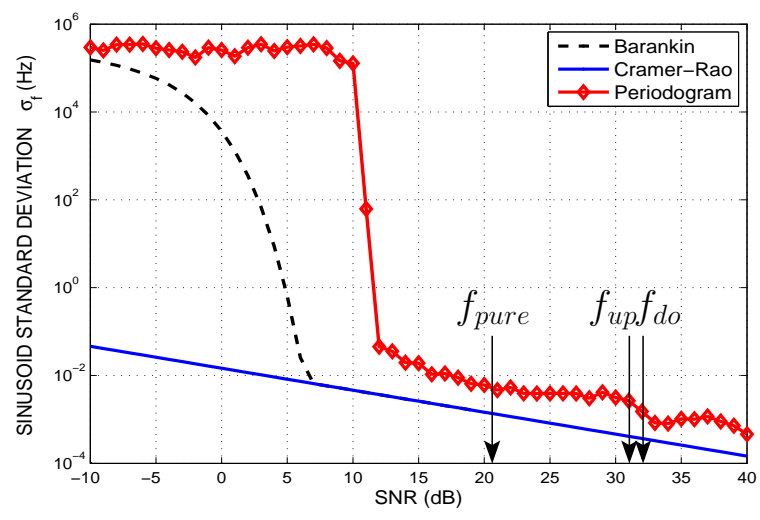

Fig. 9. Frequency estimation variance and bounds. Estimation performance for beat frequencies for a car $(d=50 \mathrm{~m}, v=80$ $\mathrm{km} / \mathrm{h})$.

\section{ADS \& Matlab platforms}

\subsection{Radar Architecture}

To promote system simplicity and keep down the unit price, the radar architecture is homodyne. The general architecture of the radar front-end is presented in Fig. 10, and Table I shows the values of the main parameters used to model the RF platform that contains the radar front-end. The $76.5 \mathrm{GHz}$ signal is generated by a 12.75 GHz Voltage-Controlled Oscillator MMIC (VCO), which is modulated according to the chosen modulation format. The VCO is followed by an MMIC including a multiplier by six, combined with a medium-power amplifier (X6MPA). At the output of the X6MPA, the chirpmodulated 76.5 GHz signal is injected into a power divider, which passes one part of the signal through a coupler to the antenna where it is to be transmitted, and the other part to the MMIC mixer to serve as the LO signal. Once the transmitted signal has passed through the 
propagation channel, it is reflected on the target (pedestrian, motorcycle, car, truck, etc.) and propagated back to the antenna. After passing through the coupler, it is amplified in a low noise amplifier (LNA), and then divided into its quadrature components (I and Q) in a second coupler. These I and Q components are finally downconverted in the MMIC mixers to generate the baseband beat frequencies.

\subsection{Modeling considerations}

The antenna and propagation channel are modelled according to the radar equation. The delay of the propagation to the target and back is also included in the propagation channel. Initially, only the line-of-sight propagation path is taken into account, and if multiple targets are present, they are considered as transparent. Thus each target is reached by a signal propagating along the lineof-sight path.

Four types of targets are considered in this study: pedestrian, motorcycle, car and truck. The model parameters are the radar cross section (RCS) and the Doppler found in simulations is about $12 \mathrm{~dB}$, which makes the 15 frequency shift associated to each target's relative velocity. Different publications of RCS measurements at $76-77 \mathrm{GHz}$, e.g. [20], [21], [22] and [23], show that the RCS of different targets must be evaluated more thoroughly. However, in this study we chose to use a fixed RCS value of $-10 \mathrm{dBsm}$ and $7 \mathrm{dBsm}$ for the pedestrian and the motorcycle, respectively. For the two larger targets, an expression obtained experimentally is used. It is given by (24) and (25) for cars and trucks respectively, and expresses the fact that at short distances the target is not completely illuminated by the antenna beam.

$$
\begin{aligned}
& R C S_{c a r}(d B)=\min \left\{\begin{array}{l}
10 \log _{10}(d)+5 \\
20
\end{array}\right. \\
& R C S_{\text {truck }}(d B)=\min \left\{\begin{array}{l}
20 \log _{10}(d)+5 \\
45
\end{array}\right.
\end{aligned}
$$

where $d$ is the distance.

\subsection{Noise modelling}

One parameter which is decisive for the choice of radar architecture is the SNR. Experiences from an earlier generation of ACC radar show that an SNR greater than $15 \mathrm{~dB}$ is necessary in order to guarantee target detection. This was also emphasised by the performance analysis of the distance and relative velocity estimation of the waveforms presented in section II D. The threshold $\mathrm{dB}$ requirement for practical operation of the radar reasonable. Thus, to obtain accurate SNRs from radar simulations, the noise of the concerned components must be modelled properly. In the front-end, the following noise sources are considered: the phase noise $(\mathrm{PN})$ of the VCO, the noise deterioration in the X6 which follows the formula $20 \log _{10}(N)$ (where $N$ is the multiplication factor), the noise figure (NF) of the MPA, the equivalent noise temperature at the antenna, the NF of 


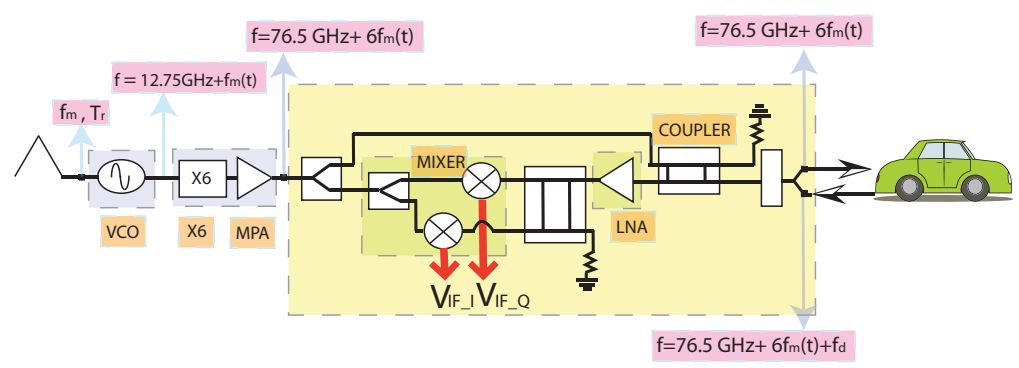

Fig. 10. The general architecture of the radar front-end, including antenna, propagation channel and target.

the LNA and finally the NF and $1 / f$ noise of the MMIC mixer.

Table 1 shows the values of the main parameters used to model the RF platform.

\subsection{ADS Implementation}

To obtain a usable simulation platform, the Advanced Design System (ADS) from Agilent Technologies was employed. ADS allows co-simulation between its builtin envelope and DSP simulators. Hence, the complete radar front-end, including the antenna, the propagation channel and the targets, is modelled using the ADS builtin elements of the analog/RF schematic. Care is taken to represent all included components as correctly as possible, according to their specifications. These RF parts are simulated using the built-in envelope simulator. The envelope simulation is launched from a baseband data flow (DF) controller that controls the flow of all mixed numeric and timed signals for all digital signal processing (DSP) simulations. The DF controller also manages the control voltage of the $\mathrm{VCO}$ where the modulation waveform is applied. The DSP level of the ADS simulation platform is presented in Fig.11. The front-end antenna propagation channel and targets (as presented in Fig.11) are included in the Analog/RF sub-system, referred to here as the "RF Platform". The I and Q baseband signals (i.e. the signal containing the beat frequencies) are collected for further signal processing, as described in section II. In the ADS-based platform, builtin spectrum analyzers are used to capture the frequency spectrum on each ramp. Unfortunately, once the spectra are available, the ADS data display offers limited possibilities for detecting, sorting and associating the peaks of each spectrum. Hence, the peak-detection-association and target-identification algorithm is almost impossible to implement. The acquired signals $V_{I F_{-} I}(t)$ and $V_{I F_{-} Q}(t)$ are therefor exported to Matlab for further processing. The radar front-end is described in more details in [24].

\subsection{Matlab Implementation}

To verify that the baseband signals generated by the ADS platform are correct, a Matlab-based platform has been developed. This platform also serves as a means to implement and test the proposed algorithms, as well as to detect and identify the targets. It also allows statistical studies. Since the baseband signal bandwidth is 
Table 1. Data of the radar front-end components.

\begin{tabular}{|c|c|c|c|}
\hline Component & \multicolumn{2}{|c|}{ Parameter } & Value \\
\hline VCO(MMIC) & $\begin{array}{r}\text { Freq } \\
\text { Outpu } \\
\text { Phase Noise }\end{array}$ & $\begin{array}{l}\text { ency } \\
\text { at } 10 \mathrm{kHz} \\
\text { at } 100 \mathrm{kHz} \\
\text { at } 1 \mathrm{MHz}\end{array}$ & $\begin{array}{c}12.75 \mathrm{GHz}+f_{m} \\
5 \mathrm{dBm} \\
-75 \mathrm{dBc} / \mathrm{Hz} \\
-100 \mathrm{dBc} / \mathrm{Hz} \\
-123 \mathrm{dBc} / \mathrm{Hz}\end{array}$ \\
\hline Multiplier-amplifier(MMIC) & $\begin{array}{r}\text { Multiplice } \\
\text { Outpu } \\
\text { Noise }\end{array}$ & $\begin{array}{l}\text { ion factor } \\
\text { power } \\
\text { igure }\end{array}$ & $\begin{array}{c}6 \\
14.5 \mathrm{dBm} \\
8 \mathrm{~dB}\end{array}$ \\
\hline LNA & $\begin{array}{r}\mathrm{G} \\
\text { Noise }\end{array}$ & n igure & $\begin{array}{l}15 \mathrm{~dB} \\
4.5 \mathrm{~dB}\end{array}$ \\
\hline Mixer(MMIC) & $\begin{array}{r}\text { Conver } \\
\text { Noise figure }\end{array}$ & $\begin{array}{l}\text { on loss } \\
\text { at } 1 \mathrm{kHz} \\
\text { at } 10 \mathrm{kHz} \\
\text { at } 100 \mathrm{kHz} \\
\text { at } 1 \mathrm{MHz}\end{array}$ & $\begin{array}{l}7.5 \mathrm{~dB} \\
34 \mathrm{~dB} \\
28 \mathrm{~dB} \\
21 \mathrm{~dB} \\
17 \mathrm{~dB}\end{array}$ \\
\hline Couplers(durod) & & ion & $\begin{array}{l}3.2 \mathrm{~dB} \\
40 \mathrm{~dB}\end{array}$ \\
\hline Transition (antenna) & & & $0.25 \mathrm{~dB}$ \\
\hline Antenna & $\begin{array}{l}\text { Maxima } \\
\text { Effective } \\
\text { Noise te }\end{array}$ & $\begin{array}{l}\text { gain }(G) \\
\text { rea }(\mathrm{RX}) \\
\text { perature }\end{array}$ & $\begin{array}{c}27 \mathrm{dBi} \\
6.06 \times 10^{-4} \mathrm{~m}^{2} \\
290 \mathrm{~K}\end{array}$ \\
\hline Propagation path & $\begin{array}{r}\text { Losses (pe } \\
\text { De }\end{array}$ & $\begin{array}{l}\text { unit area) } \\
\text { ay }\end{array}$ & $\begin{array}{c}10 \log _{10}\left(4 \pi d^{2}\right) \mathrm{dBsm} \\
\tau=d / c(\mathrm{~s})\end{array}$ \\
\hline Targets & $\begin{array}{r}\text { Doppler } \\
\operatorname{RCS}(\sigma)\end{array}$ & $\begin{array}{l}\text { equency } \\
\text { Pedestrian } \\
\text { Motorcycle } \\
\text { Car } \\
\text { Truck }\end{array}$ & $\begin{array}{c}2 v f_{0} / c(\mathrm{~Hz}) \\
-10 \mathrm{dBsm} \\
7 \mathrm{dBsm} \\
\text { see equation (24) } \\
\text { see equation (25) }\end{array}$ \\
\hline
\end{tabular}




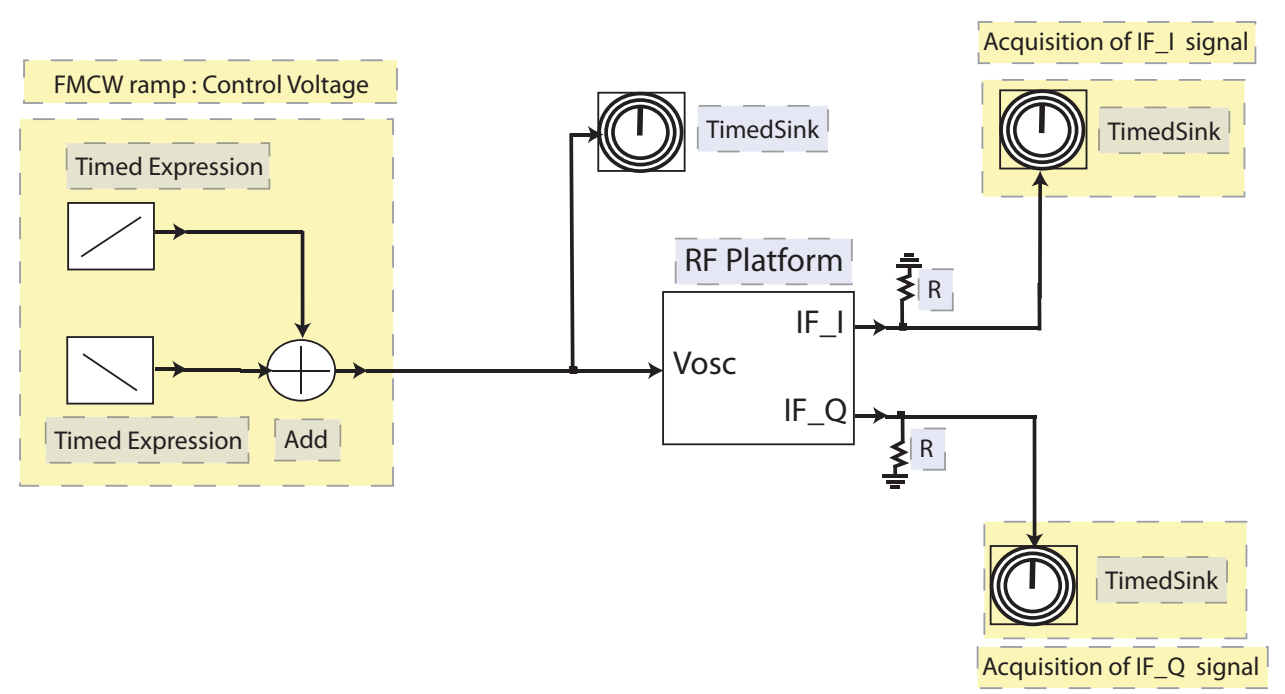

Fig. 11. General simulation platform, where the Data Flow simulation tool controls the Envelope simulation of the RF platform.

very wide $(B=600 \mathrm{MHz})$, we directly generate signals at the output of the mixer to avoid huge vector manipulation. We calculate the radar equation (26) formulated here for non-fluctuating targets, to determine the signal level for each target, taking into account all parameters in Table 1.

$$
P_{r}=P_{t} \frac{G^{2} \lambda^{2} \sigma}{(4 \pi)^{3} d^{4}}
$$

In eq (26), $P_{t}$ and $P_{r}$ are the transmitted and received powers respectively, $G$ is the gain of the radar antenna, $\lambda$ the mean wavelength of the signal, $\sigma$ the RCS and $d$ the distance to the target. The values of parameters $\lambda, G$ and $\sigma$ are those given in Table 1 .

The received signal power depends on the distance and the RCS of the targets. The mixer and VCO phase noises are calculated as in the ADS implementation, and interpolated for all frequencies. They are added in the Fourier domain of the signal: at each frequency a gaussian noise with suitable variance is added. According to the study of estimation performance, we set the detec- tion threshold for beat frequencies at $\mathrm{SNR}=15 \mathrm{~dB}$.

Beat frequencies are obtained from the local maxima of the periodogram situated above the detection threshold. Once the beat frequencies are obtained, the estimation algorithms of detection are applied using (6), (7) and (10) for dual FMCW and three-segment FMCW waveforms, respectively.

\section{Simulation results}

\subsection{Comparison of ADS and Matlab}

To compare the results of both simulation platforms (ADS and Matlab), a three-target example is set up containing a pedestrian $[15 \mathrm{~m},+80 \mathrm{~km} / \mathrm{h}]^{2}$, a motorcycle [150 $\mathrm{m},-10 \mathrm{~km} / \mathrm{h}]$ and a truck $[15 \mathrm{~m},+10 \mathrm{~km} / \mathrm{h}]$, where velocities are relative velocities between the radar and the targets. The dual FMCW and three-segment FMCW

\footnotetext{
${ }^{2} 80 \mathrm{~km} / \mathrm{h}$ is a relative speed, which means that the car is approaching the slowly moving pedestrian at about $80 \mathrm{~km} / \mathrm{h}$.
} 
modulation formats are considered with a total duration $T=5.12 \mathrm{~ms}$.

The first up ramp spectra based on ADS and Matlab simulations are shown in Fig. 12 and Fig. 13 respectively. Complex data $I+j Q$ data were used in order to enhance the visibility in the spectra. A Hamming window is used for periodogram smoothing [25]. Since complex data are used, the beat frequencies are all found at the correct side of zero in the spectrum. Using only the real part would lead to twice as many beat frequencies at both positive and negative frequencies. According to the theory, the three beat frequencies should be $-33.2 \mathrm{kHz}, 446.7 \mathrm{kHz}$ and $-43.1 \mathrm{kHz}$ for the pedestrian, motorcycle and truck, respectively. Table 2 shows the detected beat frequencies from ADS and Matlab simulations. The frequency resolution depends on the inverse of the ramp duration, leading to a precision of about $0.8 \mathrm{kHz}$ for the beat frequencies. This shows that the results in Table 2 agree perfectly with the theoretical values.

Table 2. Beat frequency comparison for the first up ramp

\begin{tabular}{|c|c|c|c|}
\hline & Pedestrian & Motorcycle & Truck \\
\hline Theory & $-33.2 \mathrm{kHz}$ & $-446.7 \mathrm{kHz}$ & $-43.1 \mathrm{kHz}$ \\
ADS & $-33.4 \mathrm{kHz}$ & $-446.8 \mathrm{kHz}$ & $-43.0 \mathrm{kHz}$ \\
Matlab & $-33.0 \mathrm{kHz}$ & $-446.5 \mathrm{kHz}$ & $-42.7 \mathrm{kHz}$ \\
\hline
\end{tabular}

Fig. 12 and Fig. 13 yield similar results for ADS and Matlab. Here the noise level has been set equal for all frequencies. It has been chosen equal to the noise level at the output of the mixer related to the beat frequency closest to the null frequency. This is done because ADS only accounts for a constant (worst case) noise level in a multitarget configuration. This is not a problem with Matlab, as shown in Fig. 14, where correlation of mixer noise is fully taken into account. Similar results are obtained with other ramps of both FMCW formats.

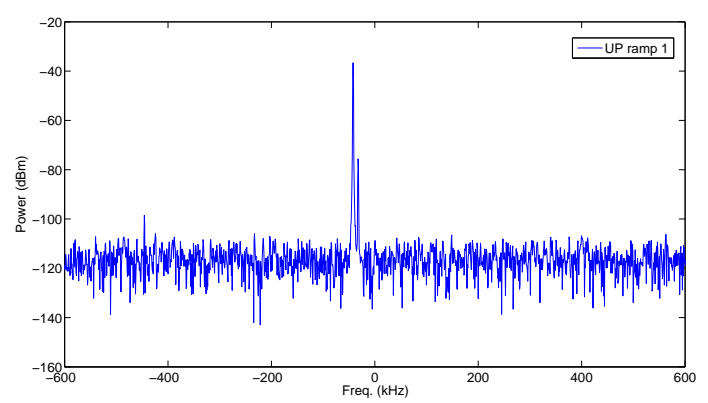

Fig. 12. ADS Spectrum for first ramp of dual FMCW waveform.

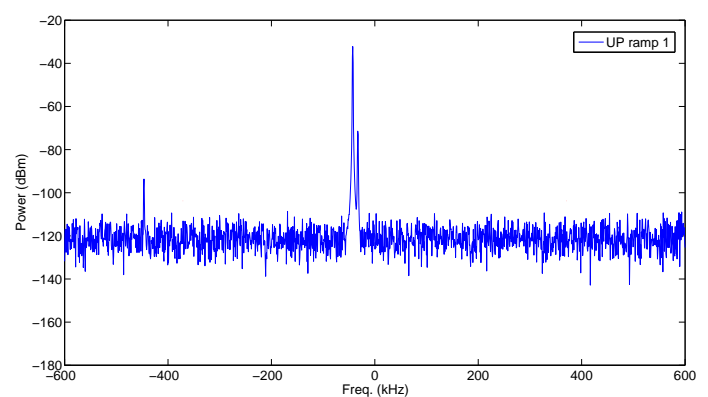

Fig. 13. Matlab Spectrum for first ramp of dual FMCW waveform.

\subsection{Target detection}

Once all spectra are calculated, the target detection algorithm is implemented and the target parameters are 


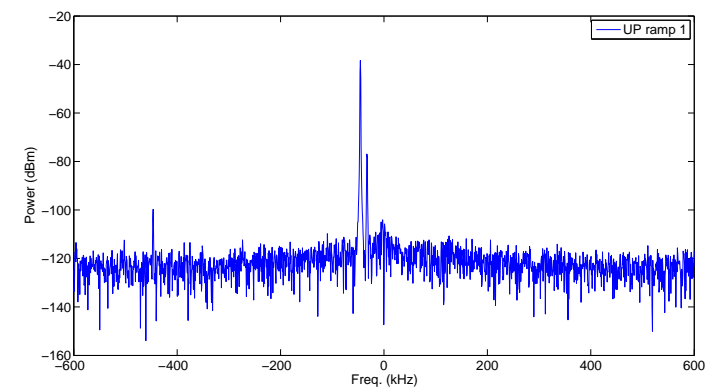

Fig. 14. Matlab Spectrum of the first ramp using dual FMCW.

All noises are taken into account.

estimated. For the dual FMCW using complex data, results are shown in Table 3.

Table 3. Results using complex data

\begin{tabular}{|c|c|c|c|c|c|}
\hline Distance & Relative velocity & RCS & SNR & Type & Detection \\
\hline 149.4 & -9.2 & 7.3 & 33.9 & Motorcycle & Yes \\
14.9 & 13.0 & 28.2 & 95.8 & Truck & Yes \\
14.9 & 82.0 & -10.4 & 56.8 & Pedestrian & Yes \\
\hline
\end{tabular}

The results obtained when using only the real part of the signal are presented in Table 4.

Table 4. Results using real part of data

\begin{tabular}{|c|c|c|c|c|c|}
\hline Distance & Relative velocity & RCS & SNR & Type & Detection \\
\hline 149.5 & -9.7 & 6.5 & 31.2 & Motorcycle & Yes \\
14.9 & 11.0 & 27.3 & 92.8 & Truck & Yes \\
14.9 & 80.0 & -11.4 & 53.8 & Pedestrian & Yes \\
\hline
\end{tabular}

When comparing Table 3 with Table 4 , it is seen that, for both the real and the complex case, all targets are detected. For both cases, the estimation of distance is excellent, but it seems that the real case returns better values for relative velocity. More generally we have tested both approaches with several examples. In some examples we obtain better results for the complex case, while the real case gives better results in other examples. Yet, it proves that the proposed algorithm works for both cases.

If the same simulation example is used for the Threesegment FMCW we will realise, that for this particular example, we have another problem, namely that the motorcycle and the truck have the same magnitude of relative velocity $(-10 \mathrm{~km} / \mathrm{h}$ and $+10 \mathrm{~km} / \mathrm{h})$. Thus, for these two targets we should find the Doppler frequencies $-1.4 \mathrm{kHz}$ and $+1.4 \mathrm{kHz}$. If using the real signal, each Doppler (and beat) frequency will turn up on both sides of zero in the spectrum. As the returned power of the truck is about $-37 \mathrm{dBm}$, while that of the motorcycle is about $-100 \mathrm{dBm}$, the former will be completely hidden by the latter. Thus, we will obtain fewer detected targets from pure Doppler frequencies. However, this will not have any effect on the final detection of targets and their distance and relative velocity estimation. Indeed, the pure Doppler frequency of the truck will enter into the algorithm as the pure frequency of the motorcycle, and it will be used together with the up and down ramp beat frequencies of the motorcycle for the calculation of $d^{\text {up }}$ and $d^{\text {down }}$ respectively. Finally the motorcycle is detected too, and its parameters are correctly estimated. Here both targets have SNRs above the detection threshold (15 dB). More generally, if the 
contributions of two targets interfere on one ramp, it is still possible to recover both of them, provided that each has a power level above the threshold on the periodograms. Note that this does not significantly degrade estimation performance, since target contributions in the periodogram are narrow and the error remains smaller than the Fourier transform resolution. In fact, it can be calculated that the resulting error on distance is less than $1 \mathrm{~m}$ and the error on relative velocity less than $1 \mathrm{~km} / \mathrm{h}$.

\section{Further experiments}

In order to show that the conclusions hold for various situations, we have considered the estimation performance bounds. We have plotted the maximum and minimum performance curves together with the mean performance curves for all $(d, v)$ couples with $d=1,50$, 100, 150, $200 \mathrm{~m}$ and $v=-180,-90,0,90,180,270$, $360 \mathrm{~km} / \mathrm{h}$. Fig. 15 and 16 clearly show that a $15 \mathrm{~dB}$ threshold above the noise level (SNR minimum) is valid for all scenarios. In addition, for SNRs larger than 15 $\mathrm{dB}$, the standard deviation is always much lower than 1 $\mathrm{m}$ for distance and $1 \mathrm{~km} / \mathrm{h}$ for relative velocity.

Each frequency of the periodogram follows an exponential distribution [26] [27]. Let us fix the detection threshold equal to $S$, and denote the variance of the noise by $\sigma^{2}$ and the periodogram output at a certain frequency by $X$. In the absence of a target, the probability of false alarm at this frequency is $P_{f a}$ :

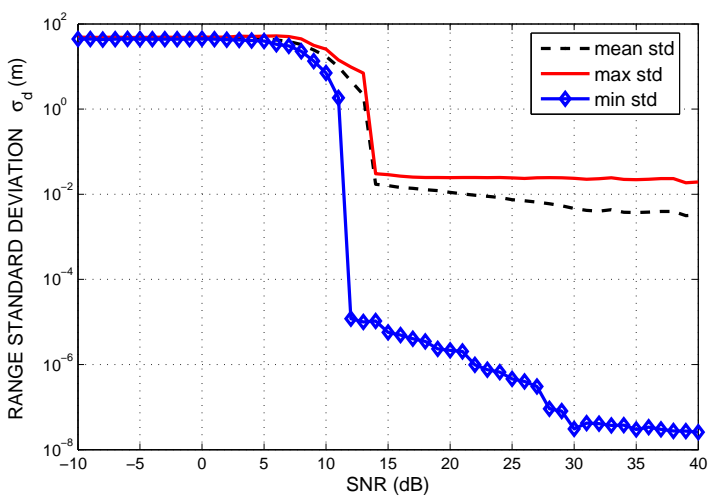

(a) distance

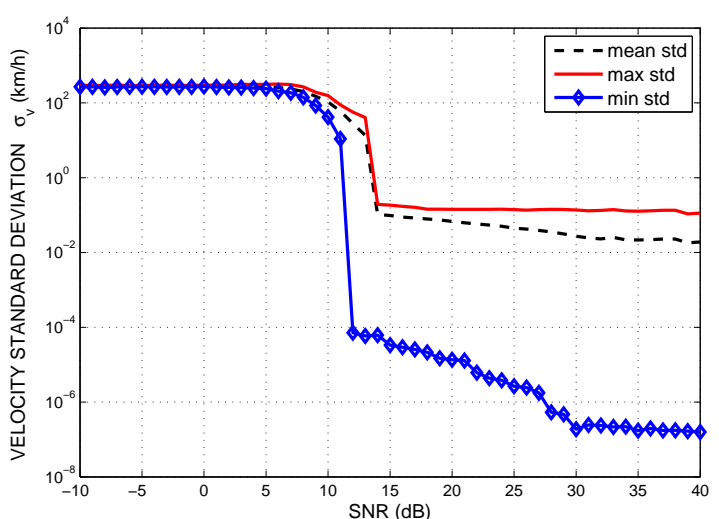

(b) Relative velocity

Fig. 15. Min, max and mean std for distance and relative velocity for dual FMCW for all 35 pairs $(\mathrm{d}, \mathrm{v})$ with $d=1,50,100,150,200 \mathrm{~m}$ and $v=-180,-90,0,90,180,270,360 \mathrm{~km} / \mathrm{h}$.

$$
\begin{aligned}
P_{f a} & =P(X>S) \\
& =\int_{S}^{\infty} \frac{1}{\sigma^{2}} e^{-\frac{u}{\sigma^{2}}} d u \\
& =\left[-e^{-\frac{u}{\sigma^{2}}}\right]_{S}^{\infty}=e^{-\frac{S}{\sigma^{2}}} \\
& =e^{-10 \frac{S_{d b}-\sigma_{d b}^{2}}{10}} .
\end{aligned}
$$

So, for all the $N$ points of the periodogram, the total false alarm probabilty is $1-\left(1-P_{f a}\right)^{N}$.

In Fig. 17 we have plotted the false alarm rate versus the threshold-to-noise ratio. We have seen before that 


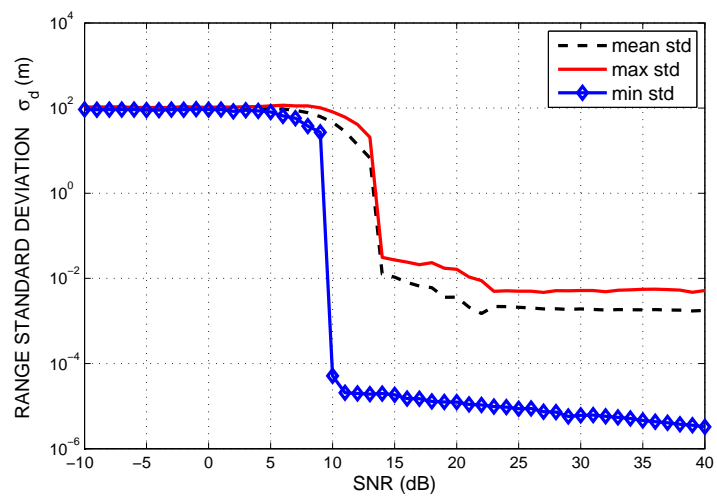

(a) distance

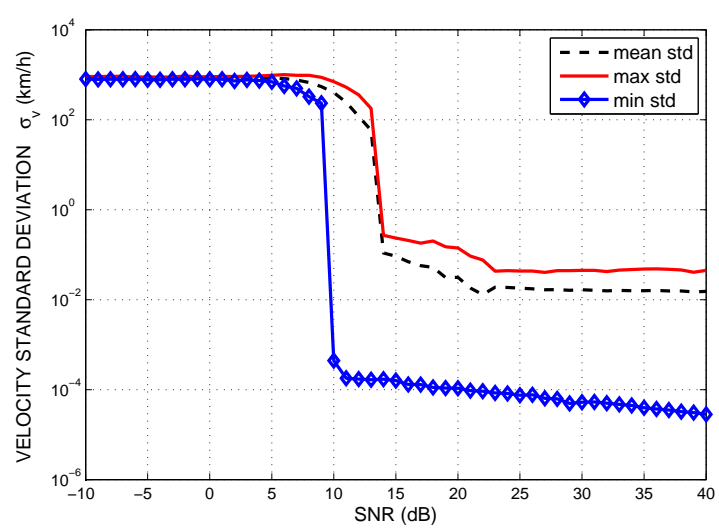

(b) Relative velocity

Fig. 16. Min, max and mean std for distance and relative velocity for three-segment FMCW for all 35 pairs $(\mathrm{d}, \mathrm{v})$ with $d=1,50,100,150,200$ and $v=$ $-180,-90,0,90,180,270,360$.

a $15 \mathrm{~dB}$ threshold above the noise level is enough for a good estimation of beat frequencies. Now, from Fig. 17 we see that with this choice, the false alarm rate is negligeable.

In order to determine if I/Q complex data or the inphase-only processing is to be preferred, we have compared the estimation standard deviation for a motorcycle and the $35(d, v)$ pairs given above. The motorcycle has been chosen since it is a target of particular interest due

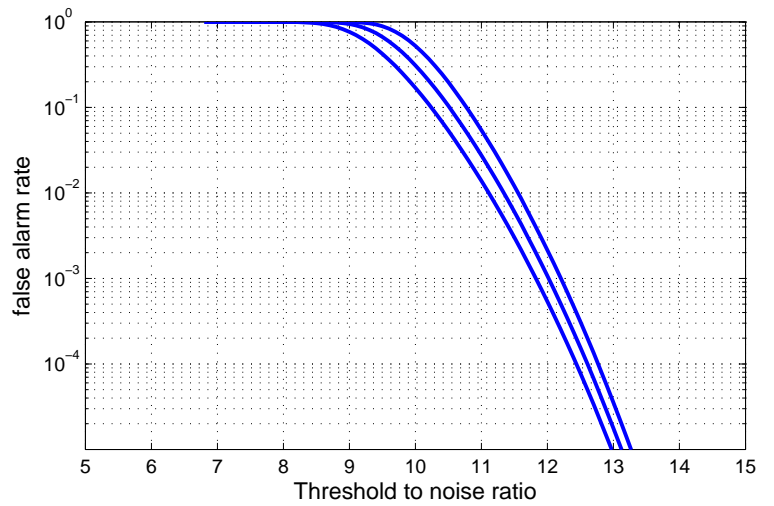

Fig. 17. False alarm rate as a function of the threshold-to-noise ratio. From left to right: 1, 2 and 4 ramps with 4096 samples per ramp.

to its low RCS, its wide relative velocity range and long distance detection requirement. The standard deviations are calculated for the estimates obtained when the target is detected. For each $(d, v)$ pair, 100 experiments have been carried out. The results are presented in tables 5 to 8. The dual FMCW outperforms the three-segment approach in most cases, but they both achieve good detection and low estimation variance. As expected, complex data processing achieves better detection due to a $3 \mathrm{~dB}$ processing loss with inphase-only processing. This results in a detection loss, as shown by the crosses $(\mathrm{X})$ in the tables.

In these tables, the first column represents situations that would lead to ambiguty when processing only inphase data with the classical FMCW (single triangle waveform). Unlike the classical FMCW waveform that shows ambiguity at short distance and hight relative speed (see section 2.1), we see that, as expected, dual and three-segment FMCW waveforms do not suffer from 
any ambiguity in the whole distance and velocity ranges.

Performance levels similar to those in tables 5 to 8 are obtained with the ADS simulation platform. This is because the same SNRs are observed for beat frequency spectra on both platforms.

Table 5. Distance and relative velocity Standard Deviation for a motorcycle when using complex data for the dual FMCW

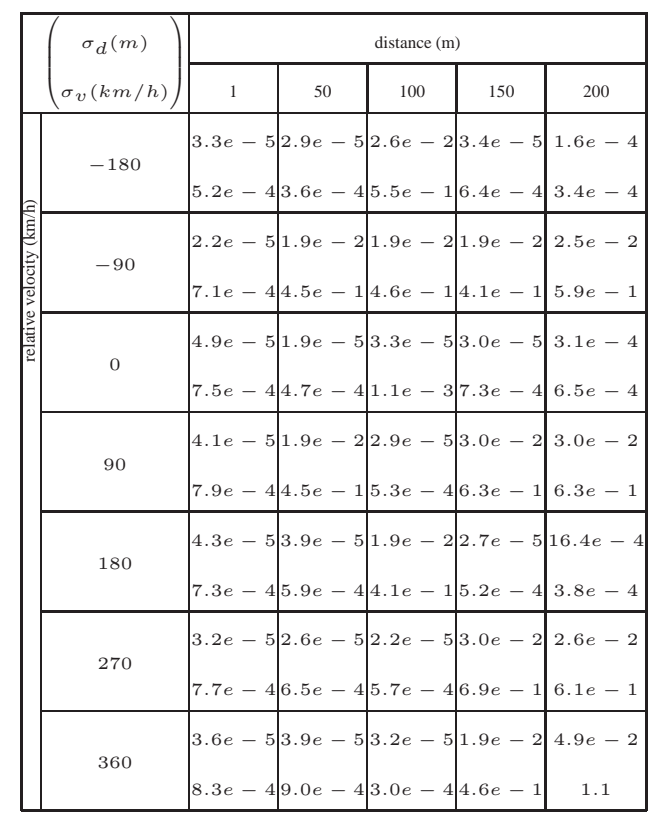

To ensure good detection of the targets, we must find the minimum transmited power $P_{t}$ that guarantees targets detection. The results are summarized in Fig. 18. We can see that $P_{t} \leq 21 \mathrm{dBm}$ in all configurations. For fixed $P_{t}$, the corresponding power density at distance $d$ from the radar antenna is

$$
\text { Power Density }=\frac{P G}{4 \pi d^{2}}
$$

where $G$ is the antenna gain. For $P_{t}=21 \mathrm{dBm}$, we get the power density as a function of $d$, plotted in Fig. 19. From this figure we can see that when a pedestrian
Table 6. Distance and relative velocity Standard Deviation for a motorcycle when using real data for the dual FMCW (X when detection is not feasible on all ramps)

\begin{tabular}{|c|c|c|c|c|c|}
\hline \multirow{2}{*}{$\left(\begin{array}{c}\sigma_{d}(m) \\
\sigma_{v}(k m / h)\end{array}\right)$} & \multicolumn{5}{|c|}{ distance $(\mathrm{m})$} \\
\hline & 1 & 50 & 100 & 150 & 200 \\
\hline-180 & $\mid \begin{array}{l}3.2 e-5 \\
4.9 e-4\end{array}$ & $\begin{array}{l}1.9 e-5 \\
4.2 e-1\end{array}$ & $\left|\begin{array}{l}2.6 e-2 \\
5.5 e-1\end{array}\right|$ & $\begin{array}{l}1.9 e-2 \\
4.1 e-1\end{array}$ & \\
\hline-90 & $\begin{array}{l}1.9 e-5 \\
4.4 e-4\end{array}$ & $\begin{array}{l}3.0 e-2 \\
6.9 e-1\end{array}$ & $\begin{array}{r}3.0 e-2 \\
7.0 e-1\end{array} \mid$ & $\begin{array}{l}x \\
x\end{array}$ & $\begin{array}{l}x \\
X\end{array}$ \\
\hline 0 & $\begin{array}{l}3.2 e-5 \\
7.8 e-4\end{array}$ & $\begin{array}{l}2.5 e-5 \\
3.0 e-4\end{array}$ & $\left|\begin{array}{l}2.1 e-5 \\
9.0 e-4\end{array}\right|$ & $\begin{array}{l}X \\
X\end{array}$ & $\begin{array}{l}x \\
X\end{array}$ \\
\hline 90 & $\begin{array}{l}2.3 e-5 \\
9.2 e-4\end{array}$ & $\begin{array}{l}3.2 e-2 \\
7.5 e-1\end{array}$ & \begin{tabular}{|}
$3.5 e-2$ \\
$6.7 e-1$
\end{tabular} \mid & $\begin{array}{l}X \\
X\end{array}$ & $x$ \\
\hline 180 & $\begin{array}{l}4.5 e-5 \\
6.9 e-4\end{array}$ & $\begin{array}{l}1.9 e-2 \\
4.1 e-1\end{array}$ & $\begin{array}{l}1.9 e-2 \\
4.2 e-1\end{array} \mid$ & $\begin{array}{l}X \\
X\end{array}$ & $x$ \\
\hline 270 & $7.7 e-4$ & $\begin{array}{l}2.4 \\
5.2\end{array}$ & $\begin{array}{l}2.6 e-5 \\
7.0 e-4\end{array} \mid$ & $\begin{array}{l}3.3 e-2 \\
7.6 e-1\end{array}$ & $X$ \\
\hline 360 & $8.4 e-4$ & $\begin{array}{l}2.6 e- \\
4.5 e-\end{array}$ & $\left|\begin{array}{l}.3 e-5 \\
4.1 e-4\end{array}\right|$ & $\begin{array}{l}1.9 e-2 \\
4.5 e-1\end{array} \mid$ & $x$ \\
\hline
\end{tabular}

Table 7. Distance and relative velocity Standard Deviation for a motorcycle when using complex data for the three-segment FMCW

\begin{tabular}{|c|c|c|c|c|c|}
\hline \multirow{2}{*}{$\left(\begin{array}{c}\sigma_{d}(m) \\
\sigma_{v}(k m / h)\end{array}\right)$} & \multicolumn{5}{|c|}{ distance (m) } \\
\hline & 1 & 50 & 100 & 150 & 200 \\
\hline-180 & $\left|\begin{array}{c}4.6 e-5 \\
7.3 e-4\end{array}\right|$ & $\begin{array}{l}1.8 e- \\
3.7 e-\end{array}$ & $\begin{array}{l}3.9 e-2 \\
6.4 e-1\end{array}$ & $\begin{array}{l}4.8 e-5 \\
1.3 e-3\end{array}$ & $\begin{array}{c}6.0 e-2 \\
0.99\end{array}$ \\
\hline-90 & $\left|\begin{array}{c}3.8 e-5 \\
7.9 e-4\end{array}\right|$ & $5.3 e-$ & $\begin{array}{r}5.8 e-5 \\
7.2 e-4\end{array}$ & $\begin{array}{l}5.9 e-5 \\
6.6 e-4\end{array}$ & $\left|\begin{array}{l}3.9 e-2 \\
6.4 e-1\end{array}\right|$ \\
\hline 0 & $\left|\begin{array}{l}5.2 e-5 \\
1.1 e-3\end{array}\right|$ & $\begin{array}{l}4.4 e- \\
7.6 e-\end{array}$ & $\begin{array}{l}4.7 e-5 \\
8.5 e-4\end{array}$ & $\begin{array}{l}1.0 e-1 \\
9.7 e-1\end{array}$ & $\left|\begin{array}{l}3.9 e-2 \\
6.5 e-1\end{array}\right|$ \\
\hline 90 & $\left|\begin{array}{l}8.3 e-5 \\
1.0 e-3\end{array}\right|$ & & $\begin{array}{r}2.3 e-5 \\
6.5 e-4\end{array}$ & $\begin{array}{l}5.3 e-2 \\
8.6 e-1\end{array}$ & $\begin{array}{l}6.7 e-5 \\
9.0 e-4\end{array} \mid$ \\
\hline 180 & $\left|\begin{array}{l}7.2 e-5 \\
1.2 e-3\end{array}\right|$ & $\begin{array}{l}3.7 e- \\
1.0 e-\end{array}$ & $\begin{array}{l}6.0 e-2 \\
9.9 e-1\end{array}$ & $\begin{array}{l}6.6 e-5 \\
1.1 e-3\end{array}$ & $\begin{array}{c}6.4 e-2 \\
1.1\end{array}$ \\
\hline 270 & $\left|\begin{array}{l}6.4 e-5 \\
1.0 e-3\end{array}\right|$ & $5.4 \mathrm{e}$ & $\begin{array}{l}5.5 e-5 \\
9.7 e-4\end{array}$ & $\begin{array}{l}3.3 e-5 \\
8.6 e-4\end{array}$ & $\begin{array}{l}3.4 e-2 \\
6.4 e-1\end{array} \mid$ \\
\hline 360 & $\begin{array}{l}2.5 e-5 \\
5.8 e-4\end{array} \mid$ & $7.3 e-$ & $\begin{array}{l}6.5 e-5 \\
1.4 e-3\end{array}$ & $\begin{array}{l}2.9 e-5 \\
5.4 e-4\end{array}$ & $\begin{array}{l}5.1 e-5 \\
6.1 e-4\end{array} \mid$ \\
\hline
\end{tabular}

is at more than $40 \mathrm{~cm}$ from the radar, the power density is below the recommended limit $\left(5 \mathrm{~mW} / \mathrm{cm}^{2}\right)$ for waves 
with frequencies between 1.5 and $100 \mathrm{GHz}$, according to the recommendation provided by the American $\mathrm{Na}$ tional Standards Intitute (ANSI) in table 1 in [28].

So, the radar complies with this norm in any situation whence there is nobody closer than $40 \mathrm{~cm}$ from it. Switching off the radar when the car is stopped or at very low speed would bring further guarantee in terms of safety.

Table 8. Distance and relative velocity Standard Deviation for a motorcycle when using real data for the three-segment FMCW (X when detection is not feasible on all ramps)

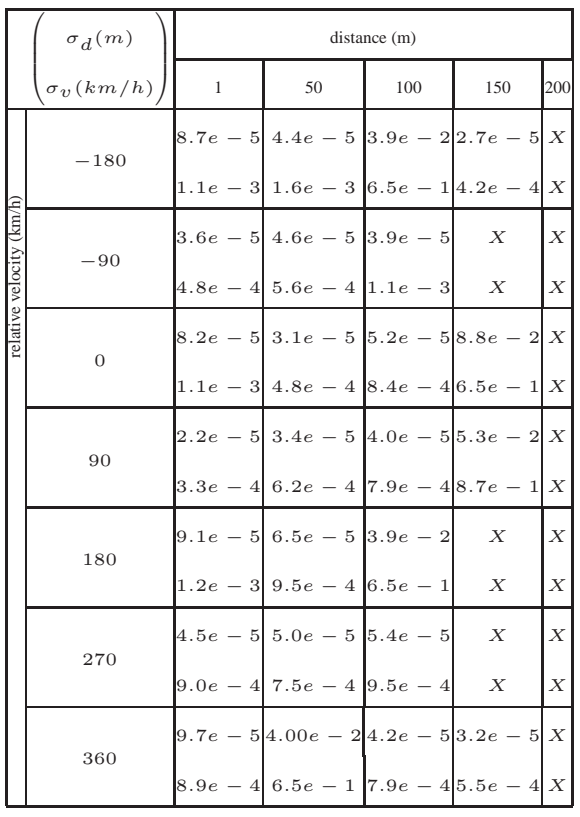

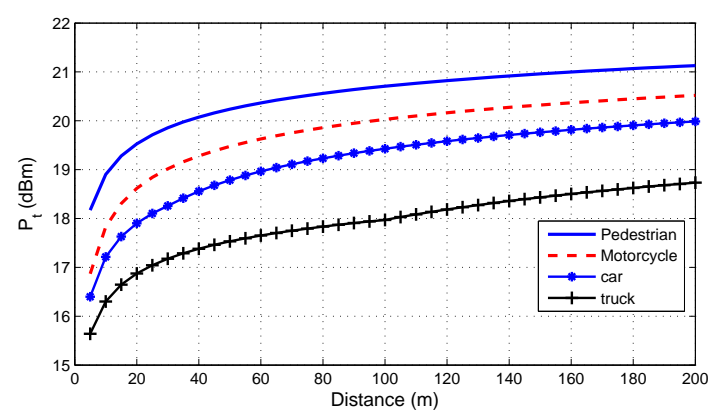

Fig. 18. Necessary transmited power to ensure good detection at distance $d$.

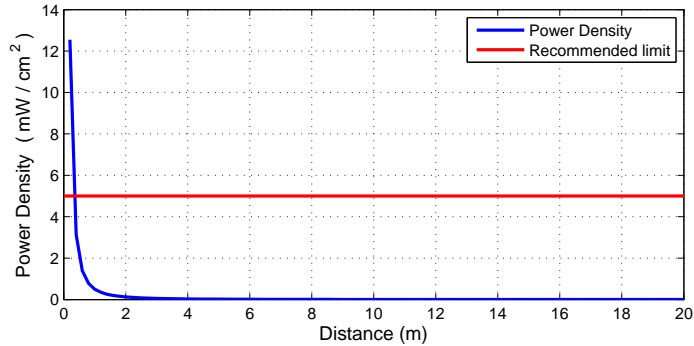

Fig. 19. Received power density at distance $d$ for transmitted power $P_{t}=21 \mathrm{dBm}$.

\section{Conclusion}

We have developed and validated two simulation platforms for a linear frequency modulation, one based on ADS and the other based on Matlab. Three modulation formats have been tested and new algorithms for distance and relative velocity estimation have been proposed. The Cramer-Rao and Barankin bounds have been used to evaluate the performance of the estimated parameters, relative velocity and distance. This validated the use of a $15 \mathrm{~dB}$ SNR threshold for target detection. It was found that the proposed dual FMCW modulation format offers slightly higher performance and low complexity in beat frequency associations compared to other strategies. The algorithms proposed allow the choice between using real or complex data; whichever is used, the targets are detected. Thus, we see that it is possible to design low complexity $77 \mathrm{GHz}$ ACC radar. This will hopefully lead to a more widespread use of ACC radars and help reduce car accident rates. 


\section{References}

1. http://epp.eurostat.ec.europa.eu/, homepage of Eurostat of the European Commission, consulted the 12 of March 2009.

2. European Commission, [Online], eSafety Compendium, Available at: http://www.esafetysupport.org/download/ eSafety_Activities/1.pdf, May 2006.

3. ETSI EN 302 288-1, Electromagnetic compatibility and Radio spectrum Matters (ERM); Short Range Devices; Road Transport and Traffic Telematics (RTTT); Short range radar equipment operating in the $24 \mathrm{GHz}$ range; Part 1: Technical requirements and methods of measurement, European Telecommunications Standards Institute, Dec. 2005.

4. ETSI TR 101983 V1.1.1, Electromagnetic compatibility and Radio spectrum Matters (ERM); Radio equipment to be used in the $76 \mathrm{GHz}$ to $77 \mathrm{GHz}$ band; System Reference Document for Short-Range Radar to be fitted on road infrastructure, European Telecommunications Standards Institute, July 2001.

5. S. Scheiblhofer, M. Treml, S. Schuster, R. Feger, and A. Stelzer, "A versatile FMCW radar system simulator for millimeter-wave applications," Proceedings of the 38th European Microwave Conference, pp. 1604-1607, Amsterdam, The Netherlands, 2008.

6. R.I. Cojocaru, E. Moldovan, B. Boukari, S. Affes, and S.O. Tatu, "A new $77 \mathrm{GHz}$ automotive phase coded CW multiport radar sensor architecture," Proceedings of 5th European Radar Conference, pp. 164-167, Amsterdam, The Netherlands, 2008.
7. H. VAN Trees, "Detection, Estimation and Modulation, Part 3," Wiley, New York, 2001.

8. E.W. Barankin, "Locally best unbiaised estimates," Ann. Math. Statist., vol. 20, pp. 447-501, 1949.

9. N. Levanon, Radar signals, Wiley, New York, 2004.

10. M.M. Meinecke, H. Rohling, "Combination of LFMCW and FSK Modulation Principles for Automotive Radar systemes," German Radar Symposium GRS 2000, Berlin, October 11-12, 2000.

11. H. Rohling, "Some Radar Topics: Waveform Design, Range CFAR and Target Recognition," Technical University Hamburg-Harburg, Hamburg, Germany, 2004.

12. J.P. Artis and S. Kemkemian, "The radar in the automotive domain," Annales des tlcommunications, vol. 60, no 3-4, pp. 326-356, 2005.

13. S. Blackman and R. Popoli, Design and Analysis of Modern Tracking System, Artech House, Norwood, MA, 1999.

14. S. Miyahara, "New Algorithm for Multiple Object Detection in the FMCW Radar," SAE Technical Paper Series , 2004-01-0177

15. S.M. Kay, "Modern Spectral Estimation - Theory and Applications," Prentice Hall, 1988.

16. D. Kok and J.S. Fu, "Signal processing for Automotive Radar," 2005 IEEE International Radar Conference, pp. 842-846, May 2005.

17. Z. Li, S.R. Lee, B. Wang and Z. Du, "Study on Reducing False Target for Automotive Radar," Proceedings on 7th International Conference on Signal Processing(ICSP '04), vol. 3, pp. $2074-2077$, Beijing, 2004.

18. L. Atallah, JP. Barbot and P. Larzabal, "From ChapmanRobbins bound towards the Barankin bound in the thresh- 
old behaviour prediction," IEEE Electronic letters, Vol. 40, N 4, pp. 279-280. 19th February 2004.

19. A. Pinkus and J. Tabrikian, "Barankin bound for range and doppler estimation using orthogonal signal transmission," IEEE Conf. on Radar, pp. 94-99, April 2006.

20. H. Suzuki, "Radar Cross Section of Automobiles for Millimeter Wave Band," JARI research Journal, Vol. 22, No. 10, pp. 475-478, 2000.

21. N. Yamada, "Radar cross section for pedestrian in 76 GHz," Proceedings of 2005 European Microwave Conference, Vol. 2, pp.46-51, 4-6 Oct. 2004,

22. R. Schneider, H.L. Blcher, K.M. Strohm, "KOKON - Automotive High Frequency Technology at 77/79 GHz,’ Proceedings of the 4th European Radar Conference, Munich, Germany, pp. 247-250, Oct. 2007.

23. K. Schuler, D. Becker, and W. Wiesbeck, "Extraction of Vertical Scattering Centers of Vehicles by Ray-Tracing Simulations," IEEE Transactions on Antennas and Propagation, Vol. 56, No. 11, pp. 3543-3551, Nov. 2008.

24. C. Krnfelt, A.Pden, A. Bazzi, G. El Haj Shhad, M. Abbas, T. Chonavel, and F. Bodereau, "77 GHz ACC Radar Simulation Platform," Proceedings of the 2009 International Transport System technology Symposium, Lille 20-22 oct. 2009.

25. A.V. Oppenheim and R.W. Schafer, "Discrete-Time Signal Processing, "Prentice-Hall, pp. 447-448, 1989.

26. T. Chonavel, "Statistical Signal Processing," Springer, 2002.

27. H. Rohling, R. Mende, "OS CFAR Performance in a 77GHz Radar Sensor For Car application,” Radar; 1996, Proceedings, CIE International Conference of, pp. 109114, Oct. 1996.
28. American National Standards Institute, "American National Standard safety levels with respect to human exposure to radio frequency electromagnetic fields, $300 \mathrm{kHz}$ to $100 \mathrm{GHz}, ”$ September 1982. 